\title{
IMPROVED HIERARCHICAL OPTIMIZATION-BASED CLASSIFICATION OF HYPERSPECTRAL IMAGES USING SHAPE ANALYSIS
}

\author{
Yuliya Tarabalka ${ }^{1,2}$ and James C. Tilton ${ }^{2}$ \\ ${ }^{1}$ INRIA, AYIN team, 2004 route des Lucioles, 06902 Sophia Antipolis, France \\ 2 NASA Goddard Space Flight Center, Greenbelt, MD 20771, USA \\ e-mail: yuliya.tarabalka@inria.fr
}

\begin{abstract}
A new spectral-spatial method for classification of hyperspectral images is proposed. The HSegClas method is based on the integration of probabilistic classification and shape analysis within the hierarchical step-wise optimization algorithm. First, probabilistic support vector machines classification is applied. Then, at each iteration two neighboring regions with the smallest Dissimilarity Criterion (DC) are merged, and classification probabilities are recomputed. The important contribution of this work consists in estimating a DC between regions as a function of statistical, classification and geometrical (area and rectangularity) features. Experimental results are presented on a 102-band ROSIS image of the Center of Pavia, Italy. The developed approach yields more accurate classification results when compared to previously proposed methods.
\end{abstract}

Index Terms - Classification, segmentation, hyperspectral images, geometrical features, rectangularity.

\section{INTRODUCTION}

The recent advances in hyperspectral remote sensor technology makes it possible to acquire data with a very high spectral (hundreds of spectral channels) and spatial (order of a meter) resolution. The rich spectral information of the hyperspectral data allow to discriminate different physical substances, leading to the potential of a more accurate classification [1]. However, when classifying hyperspectral images, two major challenges must be addressed: 1) data processing in a highdimensional spectral space [2]; 2) extraction and analysis of spatial information $[3,4]$.

In previous works, we have proposed to apply a segmentation procedure to a hyperspectral image in order to use the identified regions as pixels' spatial neighborhoods $[4,5]$. Recently, we developed a spectral-spatial classification method, which concurrently performs best merge region growing and classification, yielding accurate classification maps [5]. In this method, the decision to merge regions at each iteration was based on mean spectral vectors, sizes and classification probabilities of the corresponding regions.
In this work, we aim to further improve hierarchical model for hyperspectral image classification. For this purpose, we propose to include shape features in the region model. Analysis of shape features for object classification in remotely sensed images has been previously investigated in $[6,7,8]$. In all these works, segmentation was first performed, and then shape features of the detected regions, such as elongation, rectangularity, compactness, were analyzed. We propose to analyze shape features during the construction of a segmentation map, thus seeking for more accurate segmentation results. Thus, a new method for improved spectralspatial classification of hyperspectral data is proposed in this paper. For a given hyperspectral image, probabilistic Support Vector Machines (SVM) classification is first applied. Then, best merge region growing is performed, by iteratively merging neighboring regions with the smallest Dissimilarity Criterion (DC) and recomputing classification probabilities. The important novelty consists in adding shape analysis for computing DCs. In this paper, we include rectangularity feature for driving the segmentation process.

The paper is organized as follows. In the next section, we present a new Hierarchical Segmentation with integrated Classification (HSegClas) approach. Experimental results are described in Section 3. Finally, conclusions are drawn in Section 4.

\section{PROPOSED CLASSIFICATION METHOD}

On the input a $B$-band hyperspectral image is given, which can be considered as a set of $n$ pixel vectors $\mathbf{X}=\left\{\mathbf{x}_{j} \in\right.$ $\left.\mathbb{R}^{B}, j=1,2, \ldots, n\right\}$. The aim is to compute a classification map $\mathbf{L}=\left\{L_{j}, j=1,2, \ldots, n\right\}$, where each pixel $\mathbf{x}_{j}$ is assigned to one of $K$ thematic classes, i.e., has a class label $L_{j}$. The proposed HSegClas method consists of two main steps:

\subsection{Probabilistic pixelwise classification}

First, a pixelwise classification of the input hyperspectral image is performed, resulting in a classification map $\mathbf{L}=\left\{L_{j}, j=1,2, \ldots, n\right\}$, where each pixel has a unique class label, and class probabilities for each pixel $\left\{P\left(L_{j}=\right.\right.$ 


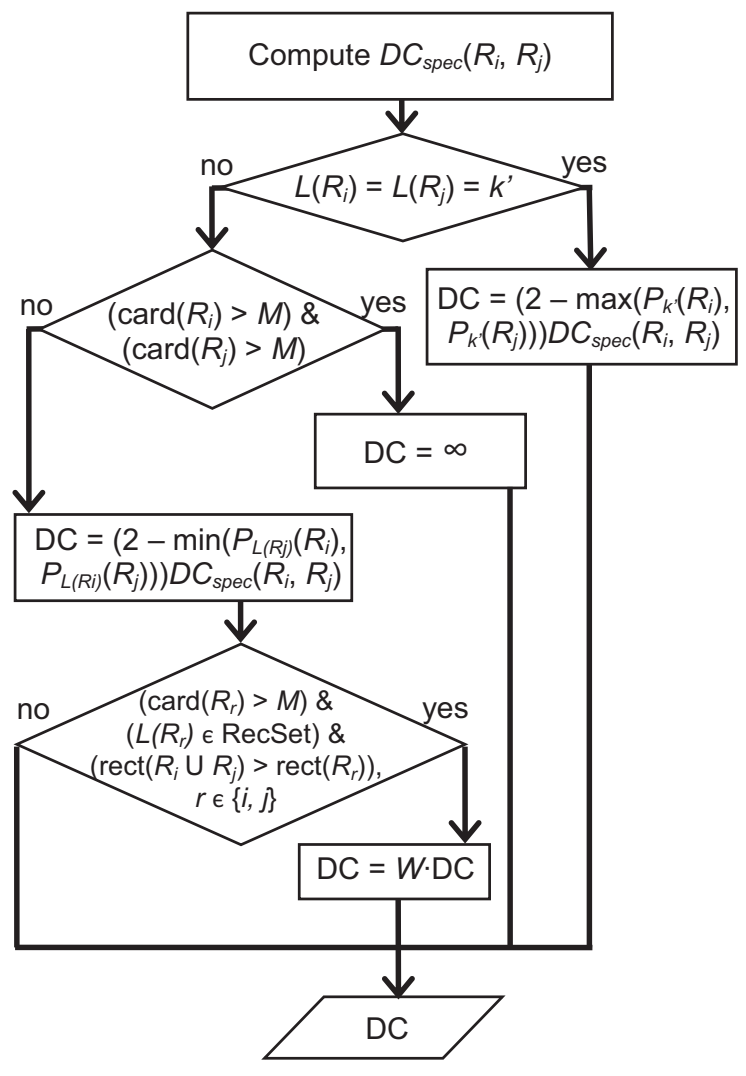

Fig. 1. Flowchart of computing a DC between two regions $R_{i}$ and $R_{j}$. "RectSet" means a set of labels for classes which represent objects with high rectangularity values.

$\left.\left.k \mid \mathbf{x}_{j}\right), k=1, \ldots, K\right\}, j=1,2, \ldots, n$. We propose to use an SVM classifier, which is well suited for classifying highdimensional data [1]. We refer the reader to [4] for details on how class probabilities are estimated using pairwise coupling of binary probability estimates.

\subsection{Hierarchical step-wise optimization}

At the next step, Hierarchical Step-Wise Optimization (HSWO) segmentation with integrated classification is performed using the following procedure:

1) Assign a new region label for each pixel. Each new region $R_{i}$ gets a preliminary class label $L\left(R_{i}\right)$ and a $K$-dimensional vector of class probabilities $\left\{P_{k}\left(R_{i}\right)=\right.$ $\left.P\left(L\left(R_{i}\right)=k \mid R_{i}\right), k=1, \ldots, K\right\}$.

2) Calculate the dissimilarity criterion $D C\left(R_{i}, R_{j}\right)$ between all pairs of spatially adjacent regions $\left\{R_{i}, R_{j}\right\}$, using an eight-connectivity neighborhood (see Fig. 1):

- First, the spectral dissimilarity $D C_{\text {spec }}\left(R_{i}, R_{j}\right)$ between two regions is estimated by computing Spectral
Angle Mapper (SAM) between the region mean vectors $\mathbf{u}_{i}=\left(u_{i 1}, \ldots, u_{i B}\right)^{T}$ and $\mathbf{u}_{j}=\left(u_{j 1}, \ldots, u_{j B}\right)^{T}$ [9]:

$$
\begin{gathered}
D C_{\text {spec }}\left(R_{i}, R_{j}\right)=S A M\left(\mathbf{u}_{i}, \mathbf{u}_{j}\right)= \\
\arccos \left(\frac{\sum_{b=1}^{B} u_{i b} u_{j b}}{\left[\sum_{b=1}^{B} u_{i b}^{2}\right]^{1 / 2}\left[\sum_{b=1}^{B} u_{j b}^{2}\right]^{1 / 2}}\right) .
\end{gathered}
$$

- If the regions have equal class labels $L\left(R_{i}\right)=L\left(R_{j}\right)=$ $k^{\prime}$,

$$
\begin{gathered}
D C\left(R_{i}, R_{j}\right)= \\
\left(2-\max \left(P_{k^{\prime}}\left(R_{i}\right), P_{k^{\prime}}\left(R_{j}\right)\right)\right) D C_{\text {spec }}\left(\mathbf{u}_{i}, \mathbf{u}_{j}\right) .
\end{gathered}
$$

- If $L\left(R_{i}\right) \neq L\left(R_{j}\right)$, analyze region size: if a number of pixels in each region is larger than $M, D C\left(R_{i}, R_{j}\right)=$ $\infty$ (the upper maximum value of $f$ loat), otherwise:

$$
\begin{gathered}
D C\left(R_{i}, R_{j}\right)= \\
\left(2-\min \left(P_{L\left(R_{j}\right)}\left(R_{i}\right), P_{L\left(R_{i}\right)}\left(R_{j}\right)\right)\right) D C_{\text {spec }}\left(\mathbf{u}_{i}, \mathbf{u}_{j}\right) .
\end{gathered}
$$

Furthermore, for adjacent regions with non-equal class labels, their rectangularity features are analyzed. A region rectangularity $\operatorname{rect}(\cdot)$ is defined as a ratio of the region area and the area of a minimum area rectangle of an arbitrary orientation including the region, and has values in the range $(0,1]$. A set of classes which represent objects with high rectangularity values is previously selected. We denote by "RecSet" a set of labels for such classes. Then, if

$$
\begin{gathered}
\left(\left(\operatorname{card}\left(R_{r}\right)>M\right) \&\left(L\left(R_{r}\right) \in \operatorname{RecSet}\right) \&\right. \\
\left(\operatorname{rect}\left(R_{i} \cup R_{j}\right)>\operatorname{rect}\left(R_{r}\right)\right)=\operatorname{true}, r \in\{i, j\}, \\
\text { then } D C\left(R_{i}, R_{j}\right)=W \cdot D C\left(R_{i}, R_{j}\right),
\end{gathered}
$$

where $\operatorname{card}(R)$ is a number of pixels in the region $R, W$ is a user set parameter, $W<1$. This means that if:

- a class label of one of the regions under consideration belongs to the RecSet set

- a size of this region is larger than $M$ pixels,

- and the rectangularity value of this region increases after it is merged with another region under consideration, the DC between these two regions is reduced by a constant multiple. Once the DC between all pairs of neighboring regions are computed, the proposed algorithm proceeds as follows:

3) Find the smallest $\mathrm{DC}$ value $D C_{\min }$.

4) Merge all pairs of neighboring regions with $D C=$ $D C_{\text {min }}$. For each new region $R_{\text {new }}=R_{i} \cup R_{j}$ recompute:

- A vector of new class probabilities as

$$
P_{k}\left(R_{\text {new }}\right)=\frac{P_{k}\left(R_{i}\right) \operatorname{card}\left(R_{i}\right)+P_{k}\left(R_{j}\right) \operatorname{card}\left(R_{j}\right)}{\operatorname{card}\left(R_{\text {new }}\right)},
$$

$k=1, \ldots, K, \operatorname{card}\left(R_{\text {new }}\right)=\operatorname{card}\left(R_{i}\right)+\operatorname{card}\left(R_{j}\right)$. 
Table 1. Results for the Center of Pavia data set. Information Classes, Number of Labeled Samples (No of samples), Global and Class-Specific Accuracies in Percentage. The Highest Accuracies are Bolded in Each Category.

\begin{tabular}{l||c||c|c|c|c|c}
\hline & No of samples & SVM & SVMMSF & HSWO+MV & HSwC & HSegClas \\
\hline Overall accuracy & - & 94.96 & 91.31 & 96.67 & 96.89 & $\mathbf{9 7 . 1 2}$ \\
Average accuracy & - & 92.56 & 92.64 & 95.41 & 96.41 & $\mathbf{9 7 . 0 3}$ \\
$\kappa$ & - & 93.61 & 89.11 & 95.77 & 96.06 & $\mathbf{9 6 . 3 5}$ \\
\hline Water & 12734 & 98.12 & 87.99 & $\mathbf{9 7 . 7 6}$ & 97.62 & 97.62 \\
Trees & 2405 & 90.48 & 89.14 & 92.80 & $\mathbf{9 3 . 6 4}$ & $\mathbf{9 3 . 6 4}$ \\
Meadows & 1788 & 94.08 & 93.52 & 91.30 & $\mathbf{9 5 . 3 9}$ & $\mathbf{9 5 . 3 9}$ \\
Bricks & 2140 & 79.86 & 87.87 & 87.96 & 91.09 & $\mathbf{9 2 . 8 9}$ \\
Bare soil & 4677 & 97.12 & 98.00 & 97.96 & $\mathbf{9 9 . 0 1}$ & $\mathbf{9 9 . 0 1}$ \\
Asphalt & 4844 & 93.52 & 93.04 & $\mathbf{9 9 . 0 7}$ & 96.07 & 96.05 \\
Bitumen & 972 & 82.48 & 92.57 & 93.21 & 95.97 & $\mathbf{9 9 . 5 8}$ \\
Tile & 1112 & 97.41 & $\mathbf{1 0 0}$ & 98.61 & 98.89 & 99.08 \\
Shadows & 2020 & 99.95 & 91.61 & $\mathbf{1 0 0}$ & $\mathbf{1 0 0}$ & $\mathbf{1 0 0}$ \\
\hline
\end{tabular}

- Class label as

$$
L\left(R_{\text {new }}\right)=\arg \max _{k}\left\{P_{k}\left(R_{\text {new }}\right)\right\} .
$$

5) Stop if each image pixel has been involved at least once in the region merging procedure. Otherwise, update the DC values between the new regions and all regions spatially adjacent to them, and go to step 3. This convergence criterion assumes that the image does not contain one-pixel objects. If it does, the algorithm may converge when $[(1-P) n]$ pixels get involved in region merging, where $P(0<P<1)$ is a probability of occurrence of one-pixel regions in the considered image.

\section{EXPERIMENTAL RESULTS AND DISCUSSION}

We applied the proposed approach to the Center of Pavia image acquired by the ROSIS (Reflective Optics System Imaging Spectrometer) sensor over the city of Pavia, Italy. The image is of 785 by 300 pixels, with a spatial resolution of 1.3 $\mathrm{m} /$ pixel and 102 spectral channels. Nine information classes are considered, which are detailed in Table 1, with the number of labeled samples for each class. Fig. 2 shows a three-band false color image and the reference data. Thirty samples for each class were randomly chosen from the reference data as training samples. The remaining samples composed the test set.

A probabilistic classification of the considered image was applied using the multiclass one versus one SVM classifier with the Gaussian radial basis function kernel and parameters chosen by five-fold cross validation: $C=128$ and $\gamma=2^{-5}$. Then, the proposed hierarchical optimization was performed, with the empirically set parameters $M=30$ and $W=0.8$. The RecSet $=\{7,8\}$ was composed of labels for classes "bitumen" and "tiles", representing rectangular roofs.

Table 1 gathers global (overall, average accuracies and kappa coefficient [10]) and class-specific accuracies of the SVM classification and the proposed HSegClas method. In order to compare the efficiency of the proposed approach with previously proposed methods, we have included results of classification using the construction of a minimum spanning forest from the probabilistic SVM-derived markers and using the SAM DC (SVMMSF) [4], which is a recently proposed and promising method. Furthermore, we have included accuracies of the classification by majority voting within the neighborhoods defined by HSWO segmentation with the SAM DC (HSWO+MV method) [11]. The optimal hierarchical level was selected by quantitatively evaluating classification accuracies of the considered method at several hierarchical levels versus the test data and retaining the best results. We also included results of the $H S w C$ approach [5], which is similar to the HSegClas method, but does not include shape analysis. Figure 2(c-e) shows some of the corresponding classification maps.

It can be seen from Table 1 that the proposed HSegClas yields the highest global and most of class-specific accuracies. It is important to note that when comparing the $\mathrm{HSwC}$ and HSegClas results, accuracies for both classes "bitumen" and "tile" are improved when applying rectangularity analysis in the new HSegClas approach, while accuracies for other classes are similar. This proves the interest of including shape features in the region model for hierarchical segmentation and classification.

\section{CONCLUSIONS}

Hyperspectral imagery enables accurate remote sensing data classification, but also presents challenges of highdimensional data analysis. In this paper, we have proposed a new HSegClas method for spectral-spatial classification of hyperspectral images. The method consists in applying probabilistic SVM classification, followed by iterative best merge region growing with integrated classification. The main novelty lies in the definition of a DC between neighboring regions as a function of region statistical, geometrical (area and rectangularity) and classification features. Experi- 


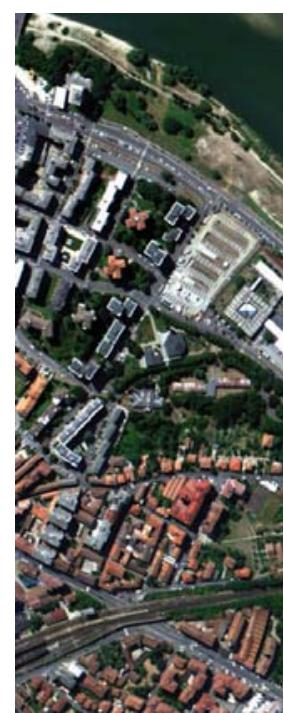

(a)

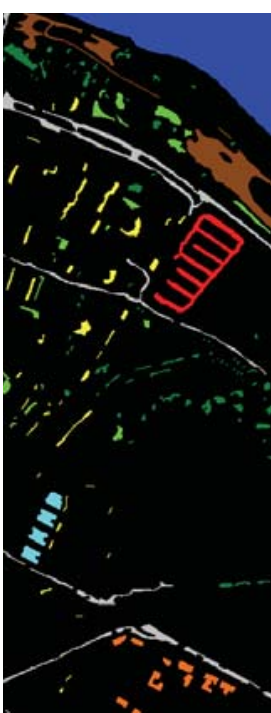

(b)

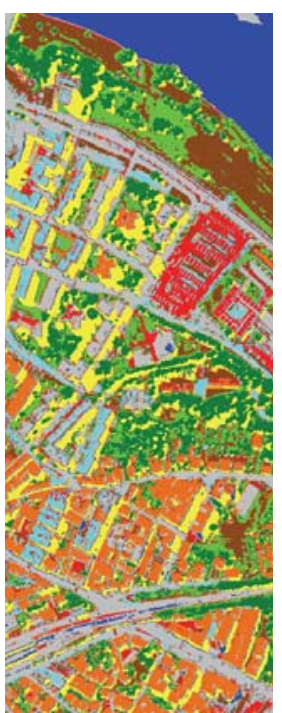

(c)

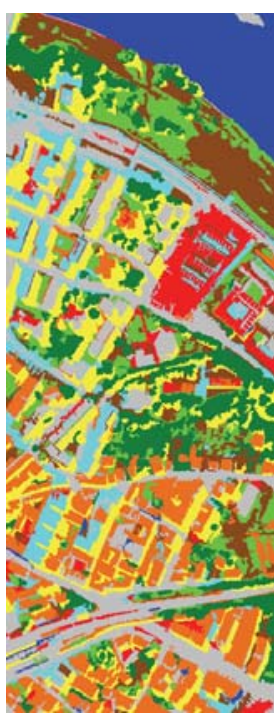

(d)

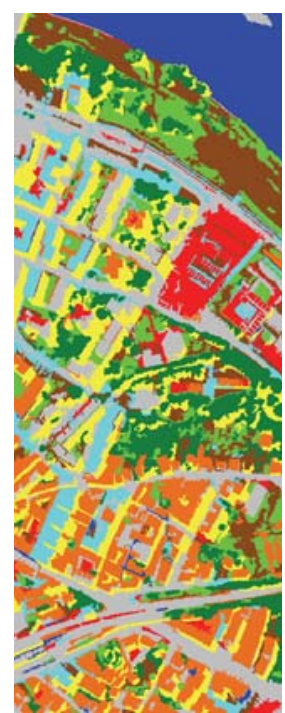

(e)

Fig. 2. Center of Pavia image. (a) Three-band color composite. (b) Reference data. (c) SVM classification map. (d) HSwC classification map. (e) HSegClas classification map.

mental results did show that the proposed method succeeded in taking advantage of both spatial and spectral information for accurate hyperspectral image classification.

\section{REFERENCES}

[1] G. Camps-Valls and L. Bruzzone, "Kernel-based methods for hyperspectral image classification," IEEE Trans. Geos. and Remote Sens., vol. 43, no. 6, pp. 1351-1362, June 2005.

[2] G. Hughes, "On the mean accuracy of statistical pattern recognizers," IEEE Trans. Information Theory, vol. 14, no. 1, pp. 55-63, Jan 1968.

[3] X. Huang and L. Zhang, "A comparative study of spatial approaches for urban mapping using hyperspectral ROSIS images over Pavia city, northern Italy," Int. Journal of Remote Sens., vol. 30, no. 12, pp. 3205-3221, 2009.

[4] Y. Tarabalka, J. Chanussot, and J. A. Benediktsson, "Segmentation and classification of hyperspectral images using minimum spanning forest grown from automatically selected markers," IEEE Trans. Systems, Man, and Cybernetics: Part B, vol. 40, no. 5, pp. 1267-1279, Oct. 2010.

[5] Y. Tarabalka and J. C. Tilton, "Best merge region growing with integrated probabilistic classification for hyperspectral imagery," in Proc. of IGARSS'11, Vancouver, Canada, 2011, pp. 3724-3727.
[6] A. K. Shackelford and C. H. Davis, "A hierarchical fuzzy classification approach for high-resolution multispectral data over urban areas," IEEE Trans. Geos. and Remote Sens., vol. 41, no. 9, pp. 1920 - 1932, Sep. 2003.

[7] M. Bouziani, K. Goita, and H. Dong-Chen, "Rulebased classification of a very high resolution image in an urban environment using multispectral segmentation guided by cartographic data," IEEE Trans. Geosc. and Remote Sens., vol. 48, no. 8, pp. 3198-3211, Aug. 2010.

[8] S. Valero, P. Salembier, J. Chanussot, and C. M. Cuadras, "Improved binary partition tree construction for hyperspectral images: application to object detection," in Proc. of IGARSS'11, Vancouver, Canada, 2011.

[9] J. C. Tilton, "RHSEG users manual: Including HSWO, HSEG, HSEGExtract, HSEGReader and HSEGViewer, version 1.50," available via email request to James.C.Tilton@nasa.gov, 2010.

[10] J. A. Richards and X. Jia, Remote Sensing Digital Image Analysis: An Introduction, Springer-Verlag New York, Inc., 1999.

[11] Y. Tarabalka, J. A. Benediktsson, J. Chanussot, and J. C. Tilton, "Multiple spectral-spatial classification approach for hyperspectral data," IEEE Trans. on Geoscience and Remote Sensing, vol. 48, no. 11, pp. 4122-4132, Nov. 2010. 